\title{
Food composition of fruits in Turkey: research outputs from the new Turkish Food Composition Database, TürKomp
}

\author{
Gül Biringen Löker ${ }^{1, \star}$, Mustafa Yaman ${ }^{1}$, Senem Akkuş Çevikkalp ${ }^{1}$, Nuran Başaran², Çağrı Özalp ${ }^{2}$, \\ Şebnem Gelgel Ünlï̈ ${ }^{2}$, Demet Demirci Gültekin ${ }^{3}$, Savaş Çalık ${ }^{3}$, Murat Öztürk ${ }^{3}$, Hasan Altay ${ }^{4}$, Ilknur \\ Türker $^{4}$, Hacer Ceyhan ${ }^{4}$, Azize Alayli Güngör ${ }^{3}$ and Birdem Amoutzopoulos ${ }^{1}$ \\ 1 TÜBİTAK Marmara Research Centre, Food Institute, Gebze, Kocaeli, Turkey \\ 2 T.R. Health Ministry, Public Health Laboratory, Istanbul No 1, Turkey \\ 3 T.R. Health Ministry, Public Health Laboratory, Erzurum, Turkey \\ 4 T.R. Health Ministry, Public Health Laboratory, Samsun, Turkey
}

Received 22 May 2015 - Accepted 4 July 2016

\begin{abstract}
In this study, both macro- and micro-nutrients of a total of 18 regional fruits were analyzed in order to launch a new and reliable food composition database (FCDB) of Turkish food composition, TürKomp. The new high quality analytical data were compared with some well-known FCDBs datasets. The new data produced in TürKomp is expected to be an invaluable source that would be used in dietary programmes and nutrition surveys in Turkey.
\end{abstract}

Keywords: Turkey / temperate and Mediterranean fruits / stone and pome fruits / nutritional value / dietary guidelines / primary and secondary nutrients

Résumé - Composition nutritionnelle des fruits de Turquie : Résultats scientifiques issus de la nouvelle table de composition alimentaire turque, TürKomp. Dans cette étude, les micro et macronutriments d'un total de 18 fruits régionaux ont été analysés dans le but d'établir une base de données alimentaires (FCDB) nouvelle et fiable, TürKomp, sur la composition nutritionnelle du régime alimentaire turc. Les nouvelles données analytiques de haute qualité ont été comparées à l'ensemble des données nationales bien connues (bases de données FCDB). Les nouvelles données produites dans TürKomp doivent apporter une information inestimable utile aux programmes alimentaires et aux enquêtes nutritionnelles en Turquie.

Mots clés : Turquie / fruits tempérés et méditerranéens / fruits à noyau et à pépins / valeur nutritionnelle / directives diététiques / micro et macro nutriments

\section{Introduction}

Turkey has a very rich plant flora due to 3 different vegetation areas (Irano-Turanien, Mediterranean and EuroSiberian) where it is situated. There are about 12,000 plant species reported to be growing in Turkey as an effect of geomorphological and climate variability. About 4,000 of them are endemic. Plant biodiversity in Turkey represents the characteristics of a continent rather than a country [1]. Agriculture plays an important role in the economy of a country. Fruits and fruit products constitute one of the major items exported by Turkey. According to the 2013 annual report of the Turkish Statistics Institute (TUIK), approximately $18 \mathrm{Mt}$ fruits have been produced in Turkey [2]. Fruit is a good source of watersoluble fiber, which is beneficial to health [3]. Fruits have an

\footnotetext{
^ Corresponding author: lokergul@gmail.com
}

important role in healthy nutrition with their high contents of K, vitamin C, and carotenoids. Apple (Malus domestica), pear (Pyrus communis), medlar (Mespilus germanica), quince (Cydonia oblonga), loquat (Eriobotrya japonica) and peach (P. persica), plum (Prunus domestica), apricot and wild apricots (Prunus armeniaca), cherries (P. avium) and sour cherries (P. cerasus), cornel (Cornus officinalis), oleaster (Eleagnus angustifolia) are the staple fruits produced and consumed in Turkey [4]. According to TBSA 2010 results, daily consumption of fresh fruits (except citrus fruits) was $51.5 \%$ in total population. In pregnancy and lactation period these values were recorded as $60.0 \%$ and $51.2 \%$ respectively [5]. In pregnancy and lactation period these values were recorded as $60.0 \%$ and $51.2 \%$ respectively.

The national food composition databases (FCDBs) are important resources for agricultural practices, food trade and 
public health. A comprehensive FCDB should include a range of food items, which cover as many foods as possible in the diet of a population. In exporting countries, such as Turkey, the importance of food trade is an important factor to be considered in food prioritisation. Efforts should be made to include the foods that have economic importance in the national FCDBs [6].

In Turkey, it had not been possible to establish a FCDB until recently. The project, Determination of National Food Composition and Formation of a Widely Available and Sustainable System, was funded by The Scientific and Technological Research Council of Turkey (TUBITAK), The Public Research Grant Committee (KAMAG) of Support Program for Research Projects of Public Institutions (TARAL 1007). The Turkish FCDB, TürKomp, was launched on 9 April 2014 as being the first food composition database of Turkey. A sustainable, original and traceable system has been used for data production. TürKomp contains approximately 63,000 analytical data for more than 500 foods covering 100 core nutrients. As it was the first food composition database in Turkey, special consideration was given to include staple fresh foods that are most important to the agricultural economy and public nutrition [7]. As a strategic plan, it endeavoured to cover as many fruit varieties as possible in TürKomp. Approximately 100 raw and processed fruit items were included in the database. To the best of our knowledge, there has been, until now, no analytical study on the nutrient content of fruits produced in Turkey using a nationally representative and systematically designed approach. This study aims to fill this gap in literature through the presentation of the nutrient composition of core fresh fruits produced in Turkey. It also provides detailed information about the comprehensive food composition techniques used for the data production with a focus on the variations of nutrient composition within geographic regions.

\section{Materials and methods}

\subsection{Selection of fruits and nutrients}

The recommended FCDB methods in the literature were used in this study for the prioritization of nutrients and the selection of foods [6, 8-12] . Researchers officially asked related organisations (e.g. Ministry of Agriculture) and Universities in Turkey to suggest nutrients and fruits that were required to be included in TürKomp. The prioritisation of fruits took into consideration these recommendations and other criteria such as; the lack of nutrient data, the economic importance of fruit, fruit biodiversity, and the frequent consumption of the fruit and marketing potential. The nutrient determination was prioritised on the basis of inclusion of relevant data in the national FCDBs and the most relevant components to be analyzed for fruits [5,9]. A total of 18 fruits (stone fruits and pome/core fruits) were included in this paper. Their macro and micronutrient levels were determined (tables I-VIII). Lan- guaL thesaurus (http://www.langual.org) was used as index food description.

\subsection{Sampling and sample preparation}

The fresh fruit samples used in this study were collected from at least 3 different vegetation regions in Turkey. The vegetation regions were selected based on the production rates of each fruit. Sampling protocols were generated for each fruit liaising with fruit experts. The samples were collected according to the protocols from the vegetation areas shown in table IX. Samples were collected at the optimum-harvesting seasons ( $t a$ ble IX) between the years of 2009-12 $(n=2)$. A minimum of $1.5 \mathrm{~kg}$ fruit was sampled from each location (the total amount of the region composite $=4.5 \mathrm{~kg}$ ). The minimum number of samples in every single region composite was 6 . The minimum number of samples in each country specific composite was 18 . The vegetation regions in Turkey are presented in figure 1 and an example on the sampling plan of peach (Prunus persica $\mathrm{cv}$. J.H. Hale) is given in figure 2 .

Samples were transferred to the central laboratory in cooling boxes. Each fruit sample was prepared according to "validated sample preparation procedures' generated by the fruit experts. Samples were prepared for laboratory analysis in appropriate laboratory conditions. Fresh samples were directly processed upon arrival to the laboratory in order to prevent nutrient oxidation. First, the samples were gently cleaned with a paper towel in order to remove the dirt, afterwards the inedible parts were removed. Only the edible parts of the samples (with skin) were added to the composite (table IX). Each sample was cut into approximately $1 \mathrm{~cm}$ pieces. Samples were immediately combined and homogenized within a stainless steel food processor to generate a composite sample for each region. Then, the composite sample was dispensed into subsamples for analyses. Polyethylene sample jars wrapped with aluminium foil were used for subsample distribution and storage. The samples were stored at $-18^{\circ} \mathrm{C}$ until analysis. Prior to storage, water content of the samples was analysed.

\subsection{Composition analysis}

Detailed quality control was applied for all analyses: method validation (MV), proficiency tests (PT) attendances, analyses of commercially available standard reference materials (SRM) and testing in-house reference materials. At the beginning of the study the technical staff of the partner laboratories attended training programs on analytical MV procedures and method uncertainty (MU) calculations. The Standard Operation Procedures (SOP) were generated for each analysis method. The laboratories set up and validated the analytical methods, calculated MUs and reported data. The analyses were repeated twice. During the project, data producers participated in routine PTs. In addition, inter-laboratory checks were carried out. In order to assure and improve the data quality an internal auditing group was established. Each laboratory was monitored on-site for several criteria (e.g. condition of instruments, consumables and calibrations). The analysis data were verified on laboratory notebooks, quality control graphics, and analysis forms. The documentation was controlled on instrument analysis printouts, MV and MU reports, retention samples and PT results. 
Table I. Proximate and carbohydrate composition of plums (average values for the regions, $100 \mathrm{~g}^{-1}$ edible food).

\begin{tabular}{ccccccccccccccc}
\hline Fruits & Regions & $\begin{array}{c}\text { Water } \\
(\mathrm{g})\end{array}$ & $\begin{array}{c}\text { Enerc } \\
(\mathrm{kcal})\end{array}$ & $\begin{array}{c}\text { Ash } \\
(\mathrm{g})\end{array}$ & $\begin{array}{c}\text { Prot } \\
(\mathrm{g})\end{array}$ & $\begin{array}{c}\text { Fat } \\
(\mathrm{g})\end{array}$ & $\begin{array}{c}\text { Fibt } \\
(\mathrm{g})\end{array}$ & $\begin{array}{c}\text { Fibsol } \\
(\mathrm{g})\end{array}$ & $\begin{array}{c}\text { Fibins } \\
(\mathrm{g})\end{array}$ & $\begin{array}{c}\text { CHO } \\
(\mathrm{g})\end{array}$ & $\begin{array}{c}\text { Sucs } \\
(\mathrm{g})\end{array}$ & $\begin{array}{c}\text { Glus } \\
(\mathrm{g})\end{array}$ & $\begin{array}{c}\text { Frus } \\
(\mathrm{g})\end{array}$ \\
\hline \multirow{4}{*}{1} & Med & 84.4 & 55 & 0.39 & 0.15 & 0.26 & 1.62 & 0.58 & 1.05 & 13.2 & 0.70 & 3.13 & 2.35 & 2 \\
& $\mathrm{~A}$ & 83.3 & 61 & 0.39 & 0.13 & 0.26 & 1.37 & 0.52 & 0.86 & 14.5 & 0.47 & 6.68 & 3.64 & 3 \\
& $\mathrm{M}$ & 84.8 & 55 & 0.56 & 0.19 & 0.18 & 1.19 & 0.16 & 1.03 & 13.1 & 0.61 & 5.07 & 2.21 & 2 \\
& Mean & 84.0 & 58 & 0.44 & 0.15 & 0.23 & 1.39 & 0.43 & 0.96 & 13.7 & 0.57 & 5.20 & 2.86 & 7 \\
& $\mathrm{SD}$ & 1.9 & 5 & 0.13 & 0.05 & 0.13 & 0.39 & 0.27 & 0.30 & 2.2 & 0.72 & 2.21 & 0.91 & 7 \\
\hline & Med & 87.1 & 48 & 0.55 & 0.08 & 0.33 & 1.49 & 0.66 & 0.83 & 10.50 & 0.00 & 8.21 & 3.79 & 2 \\
& $\mathrm{~A}$ & 87.4 & 46 & 0.70 & 0.16 & 0.61 & 2.49 & 1.10 & 1.39 & 8.64 & 0.00 & 6.49 & 2.82 & 3 \\
2 & $\mathrm{M}$ & 81.2 & 71 & 0.60 & 0.25 & 0.59 & 2.50 & 1.11 & 1.39 & 14.94 & 0.10 & 8.63 & 4.99 & 2 \\
& Mean & 85.5 & 54 & 0.63 & 0.16 & 0.52 & 2.20 & 0.98 & 1.23 & 10.97 & 0.03 & 7.59 & 3.71 & 7 \\
& SD & 4.3 & 17 & 0.31 & 0.08 & 0.24 & 0.91 & 0.40 & 0.50 & 3.95 & 0.07 & 1.35 & 1.26 & 7 \\
\hline
\end{tabular}

1: European plum group Stanley, 2: Japanese plum group Black Amber,

A: Aegean, M: Marmara, Med: Mediterranean.

Mean: Mean of individual samples, SD: Standard deviation, NA: Not available.

EuroFIR/INFOODS component tagnames [13, 14]: WATER: water; ENERC: energy, total metabolisable; ASH: ash; PROT: protein; FAT: fat, total; FIBT/FIBTG: fibre, total dietary; FIBSOL: fibre, water-soluble; FIBINS: fibre, water-insoluble; CHO: carbohydrate; SUCS: sucrose; GLUS: glucose; FRUS: fructose.

Table II. Proximate and carbohydrate composition of cherries (average values for the regions, $100 \mathrm{~g}^{-1}$ edible food).

\begin{tabular}{|c|c|c|c|c|c|c|c|c|c|c|c|c|c|c|}
\hline Fruits & Regions & $\begin{array}{c}\text { Water } \\
(\mathrm{g})\end{array}$ & $\begin{array}{l}\text { Enerc } \\
\text { (kcal) }\end{array}$ & $\begin{array}{c}\text { Ash } \\
(\mathrm{g})\end{array}$ & $\begin{array}{l}\text { Prot } \\
\text { (g) }\end{array}$ & $\begin{array}{l}\text { Fat } \\
\text { (g) }\end{array}$ & $\begin{array}{c}\text { Fibt } \\
(\mathrm{g})\end{array}$ & $\begin{array}{c}\text { Fibsol } \\
\text { (g) }\end{array}$ & $\begin{array}{c}\text { Fibins } \\
(\mathrm{g})\end{array}$ & $\begin{array}{c}\mathrm{CHO} \\
(\mathrm{g})\end{array}$ & $\begin{array}{l}\text { Sucs } \\
(\mathrm{g})\end{array}$ & $\begin{array}{c}\text { Glus } \\
\text { (g) }\end{array}$ & $\begin{array}{c}\text { Frus } \\
(\mathrm{g})\end{array}$ & $n$ \\
\hline \multirow{5}{*}{1} & $\mathrm{BS}$ & 82.6 & 65 & 0.65 & 0.23 & 0.57 & 2.69 & 0.31 & 2.38 & 13.3 & 0.09 & 6.91 & 4.21 & 2 \\
\hline & A & 81.0 & 68 & 0.69 & 1.29 & 0.52 & 4.05 & 1.48 & 2.57 & 13.5 & 0.01 & 4.46 & 5.35 & 3 \\
\hline & $\mathrm{NE}$ & 83.1 & 57 & 0.72 & 0.32 & 0.13 & 4.23 & 1.30 & 2.94 & 11.5 & 0.10 & 4.72 & 3.35 & 2 \\
\hline & Mean & 82.0 & 64 & 0.69 & 0.71 & 0.42 & 3.71 & 1.09 & 2.62 & 12.9 & 0.06 & 5.24 & 4.45 & 7 \\
\hline & SD & 1.4 & 6 & 0.06 & 1.17 & 0.55 & 0.74 & 0.73 & 0.40 & 1.6 & 0.07 & 1.72 & 1.72 & 7 \\
\hline \multirow{5}{*}{2} & Med & 83.0 & 62 & 0.40 & 0.58 & 0.38 & 1.98 & 0.31 & 1.68 & 13.1 & 0.00 & 9.17 & 5.11 & 2 \\
\hline & $\mathrm{A}$ & 81.2 & 70 & 0.90 & 0.70 & 0.30 & 2.00 & 0.30 & 1.70 & 14.8 & 0.00 & 8.69 & 5.28 & 2 \\
\hline & MS & 84.9 & 56 & 0.85 & 0.75 & 0.30 & 1.64 & 0.24 & 1.41 & 11.9 & 0.02 & 7.67 & 4.61 & 2 \\
\hline & Mean & 83.0 & 63 & 0.71 & 0.69 & 0.34 & 1.89 & 0.29 & 1.61 & 13.3 & 0.01 & 8.51 & 5.00 & 6 \\
\hline & $\mathrm{SD}$ & 2.2 & 9 & 0.35 & 0.21 & 0.09 & 0.36 & 0.05 & 0.32 & 2.1 & 0.02 & 1.35 & 0.64 & 6 \\
\hline \multirow{5}{*}{3} & $\mathrm{~A}$ & 83.1 & 61 & 1.03 & 0.93 & 0.39 & 2.59 & 0.43 & 2.16 & 12.0 & 0.00 & 8.21 & 4.43 & 2 \\
\hline & $\mathrm{ME}$ & 82.8 & 65 & 0.66 & 0.83 & 0.25 & 1.27 & 0.21 & 1.07 & 14.2 & 0.00 & 8.71 & 5.58 & 2 \\
\hline & MS & 83.1 & 63 & 0.61 & 0.96 & 0.26 & 1.39 & 0.22 & 1.17 & 13.7 & 0.00 & 9.34 & 5.58 & 3 \\
\hline & Mean & 83.0 & 63 & 0.74 & 0.91 & 0.29 & 1.70 & 0.28 & 1.42 & 13.4 & 0.00 & 8.83 & 5.25 & 7 \\
\hline & SD & 0.8 & 4 & 0.21 & 0.37 & 0.09 & 0.66 & 0.11 & 0.55 & 1.3 & 0.00 & 1.07 & 0.66 & 7 \\
\hline \multirow{5}{*}{4} & $\mathrm{~A}$ & 83.3 & 58 & 0.84 & 0.65 & 0.54 & 4.21 & 0.27 & 2.62 & 10.5 & 2.10 & 0.01 & 0.04 & 2 \\
\hline & MS & 83.7 & 59 & 0.75 & 0.57 & 0.31 & 2.65 & 0.39 & 2.26 & 12.0 & 2.80 & 0.01 & 0.02 & 2 \\
\hline & $\mathrm{MN}$ & 81.9 & 65 & 0.87 & 0.53 & 0.49 & 2.61 & 0.25 & 2.36 & 13.6 & 5.90 & 0.02 & 0.02 & 2 \\
\hline & Mean & 83.0 & 61 & 0.82 & 0.58 & 0.45 & 3.16 & 0.30 & 2.41 & 12.0 & 0.02 & 8.15 & 4.68 & 6 \\
\hline & SD & 1.2 & 5 & 0.16 & 0.21 & 0.18 & 1.23 & 0.10 & 0.42 & 1.7 & 0.03 & 1.55 & 0.75 & 6 \\
\hline \multirow{2}{*}{5} & $\overline{B S}$ & 79.9 & 75 & 0.68 & 1.40 & 0.54 & 2.82 & 0.50 & 2.32 & 14.7 & 0.00 & 5.09 & 6.36 & 2 \\
\hline & Mean & 79.9 & 75 & 0.68 & 1.40 & 0.54 & 2.82 & 0.50 & 2.32 & 14.7 & 0.00 & 5.09 & 6.36 & 2 \\
\hline
\end{tabular}

1: Cornelian cherry, 2: Sweet cherry, white, 'Starks Gold', 3: Sweet cherry, red, 'Ziraat 0900', 4: Sour cherry, 'Kütahya', 5: Cherry laurel. MN: Mid,North, A: Aegean, Med: Mediterranean, BS: Black Sea, ME: Middle East, NE: North East, MS: Mid, Southern. Mean: Mean of individual samples, SD: Standard deviation, NA: Not available.

EuroFIR/INFOODS component tagnames [13, 14]: WATER: water; ENERC: energy, total metabolisable; ASH: ash; PROT: protein; FAT: fat, total; FIBT/FIBTG: fibre, total dietary; FIBSOL: fibre, water-soluble; FIBINS: fibre, water-insoluble; CHO: carbohydrate; SUCS: sucrose; GLUS: glucose; FRUS: fructose. 
Table III. Proximate and carbohydrate composition of almond, apricot, nectarine, and peach (average values for the regions, $100 \mathrm{~g}^{-1}$ edible food).

\begin{tabular}{|c|c|c|c|c|c|c|c|c|c|c|c|c|c|c|}
\hline Fruits & Regions & $\begin{array}{c}\text { Water } \\
(\mathrm{g})\end{array}$ & $\begin{array}{l}\text { Enerc } \\
(\mathrm{kcal})\end{array}$ & $\begin{array}{c}\text { Ash } \\
(\mathrm{g})\end{array}$ & $\begin{array}{c}\text { Prot } \\
(\mathrm{g})\end{array}$ & $\begin{array}{l}\text { Fat } \\
(\mathrm{g})\end{array}$ & $\begin{array}{c}\text { Fibt } \\
(\mathrm{g})\end{array}$ & $\begin{array}{c}\text { Fibsol } \\
(\mathrm{g})\end{array}$ & $\begin{array}{c}\text { Fibins } \\
(\mathrm{g})\end{array}$ & $\begin{array}{c}\mathrm{CHO} \\
(\mathrm{g}) \\
\end{array}$ & $\begin{array}{c}\text { Sucs } \\
(\mathrm{g})\end{array}$ & $\begin{array}{c}\text { Glus } \\
(\mathrm{g}) \\
\end{array}$ & $\begin{array}{c}\text { Frus } \\
(\mathrm{g}) \\
\end{array}$ & $n$ \\
\hline \multirow{5}{*}{1} & Med & 86.5 & 45 & 0.72 & 3.13 & 0.72 & 6.28 & 0.27 & 4.87 & 3.85 & 6.55 & 0.01 & 0.06 & 2 \\
\hline & A & 87.9 & 39 & 0.77 & 4.04 & 0.71 & 5.54 & 0.30 & 4.56 & 1.69 & 6.10 & 0.01 & 0.05 & 3 \\
\hline & MS & 87.1 & 42 & 0.73 & 4.20 & 0.96 & 6.17 & 0.37 & 4.07 & 2.25 & 7.50 & 0.02 & 0.08 & 2 \\
\hline & Mean & 87.3 & 41 & 0.75 & 3.82 & 0.78 & 5.93 & 0.31 & 4.51 & 2.46 & 0.02 & 0.99 & 0.69 & 7 \\
\hline & $\mathrm{SD}$ & 0.9 & 4 & 0.06 & 0.61 & 0.18 & 1.50 & 0.06 & 0.81 & 1.48 & 0.05 & 0.43 & 0.25 & 7 \\
\hline \multirow{4}{*}{2} & Med & 90.9 & 28 & 1.74 & 0.28 & 0.21 & 1.32 & 0.22 & 1.10 & 5.62 & 0.49 & 0.80 & 0.65 & 2 \\
\hline & A & 91.0 & 28 & 1.73 & 0.25 & 0.21 & 1.16 & 0.34 & 0.83 & 5.71 & 0.80 & 1.21 & 0.95 & 2 \\
\hline & Mean & 90.9 & 28 & 1.73 & 0.27 & 0.21 & 1.24 & 0.28 & 0.96 & 5.66 & 0.70 & 1.07 & 0.85 & 4 \\
\hline & SD & 0.3 & 4 & 1.24 & 0.06 & 0.03 & 0.56 & 0.24 & 0.79 & 0.73 & 0.18 & 0.29 & 0.25 & 4 \\
\hline \multirow{5}{*}{3} & $\mathrm{M}$ & 87.7 & 44 & 0.67 & 0.16 & 0.37 & 2.20 & 0.81 & 1.39 & 8.89 & 1.91 & 2.12 & 2.23 & 2 \\
\hline & Med & 88.4 & 42 & 0.62 & 0.19 & 0.34 & 2.00 & 0.73 & 1.27 & 8.46 & 3.09 & 3.74 & 3.61 & 2 \\
\hline & A & 88.3 & 43 & 0.57 & 0.27 & 0.35 & 2.01 & 0.75 & 1.27 & 8.51 & 3.06 & 3.60 & 2.27 & 2 \\
\hline & Mean & 88.1 & 43 & 0.62 & 0.20 & 0.35 & 2.07 & 0.76 & 1.31 & 8.62 & 2.68 & 3.15 & 2.70 & 6 \\
\hline & $\mathrm{SD}$ & 0.63 & 1 & 0.14 & 0.17 & 0.07 & 0.44 & 0.16 & 0.27 & 0.33 & 1.20 & 1.15 & 1.23 & 6 \\
\hline \multirow{5}{*}{4} & Med & 90.7 & 35 & 0.34 & 0.71 & 0.24 & 1.33 & 0.33 & 0.99 & 6.70 & 2.18 & 2.05 & 1.84 & 2 \\
\hline & $\mathrm{A}$ & 88.5 & 42 & 0.41 & 0.96 & 0.27 & 1.53 & 0.46 & 1.25 & 8.15 & 2.80 & 1.79 & 1.76 & 3 \\
\hline & $\mathrm{M}$ & 87.6 & 46 & 0.44 & 0.84 & 0.16 & 1.63 & 0.36 & 1.27 & 9.40 & 4.39 & 1.85 & 1.66 & 2 \\
\hline & Mean & 88.9 & 41 & 0.40 & 0.85 & 0.23 & 1.50 & 0.39 & 1.18 & 8.09 & 2.86 & 1.89 & 1.77 & 7 \\
\hline & SD & 1.57 & 6 & 0.07 & 0.34 & 0.12 & 0.22 & 0.11 & 0.26 & 1.57 & 1.87 & 0.59 & 0.39 & 7 \\
\hline \multirow{5}{*}{5} & $\mathrm{M}$ & 87.3 & 47 & 0.50 & 1.06 & 0.35 & 1.88 & 0.62 & 1.27 & 8.96 & 2.72 & 1.02 & 1.04 & 2 \\
\hline & BS & 87.9 & 45 & 0.36 & 1.02 & 0.19 & 1.72 & 0.49 & 1.24 & 8.83 & 3.39 & 1.92 & 1.75 & 2 \\
\hline & $\mathrm{A}$ & 87.3 & 47 & 0.46 & 0.81 & 0.38 & 1.86 & 0.50 & 1.36 & 9.19 & 4.03 & 1.41 & 1.51 & 2 \\
\hline & Mean & 87.5 & 46 & 0.44 & 0.96 & 0.31 & 1.82 & 0.54 & 1.29 & 8.99 & 3.38 & 1.45 & 1.43 & 6 \\
\hline & SD & 0.77 & 3 & 0.09 & 0.28 & 0.16 & 0.35 & 0.16 & 0.22 & 1.17 & 1.87 & 0.66 & 0.57 & 6 \\
\hline
\end{tabular}

1: Green almond, 2: Apricot early season 'Tyrinthe', 3: Nectarine 'Big Top', 4: Peach, early season, 5: Peach, 'J. Hale'.

A: Aegean, M: Marmara, Med: Mediterranean, BS: Black Sea, MS: Mid, Southern. Mean: Mean of individual samples, SD: Standard deviation, NA: Not available.

EuroFIR/INFOODS component tagnames [13, 14]: WATER: water; ENERC: energy, total metabolisable; ASH: ash; PROT: protein; FAT: fat, total; FIBT/FIBTG: fibre, total dietary; FIBSOL: fibre, water-soluble; FIBINS: fibre, water-insoluble; CHO: carbohydrate; SUCS: sucrose; GLUS: glucose; FRUS: fructose.

All analyses were performed in the edible portion of $100 \mathrm{~g}$ food. Energy was calculated using general Atwater factors. The energy calculation factors of protein, total fat, carbohydrate, sugar alcohols (sorbitol, mannitol, xylitol) and total dietary fiber were $4,9,4,2.4$, and $2.0 \mathrm{kcal}^{-1} \mathrm{~g}^{-1}$ respectively. Carbohydrate (available) was determined 'by difference' method $[6,15,16]$. The water content of foods were determined by calculating the water content from the loss of weight due to drying process in air-oven under a specific temperature [17-19]. Ash was determined by using ash-oven at $550 \pm 25^{\circ} \mathrm{C}$ and weighing the dry mineral residue $[20,21]$. The total protein was determined by 'Kjeldahl digestion' method and 6.25 was the conversion factor $[18,25,26]$. The total fat content was determined by Soxhlet and lipid conversion factor was 0.800 [27-29]. The total dietary fibre level was calculated by enzymatic-gravimetric analysis using MES/TRIS buffer solution [30,31]. Individual sugars were measured by HPLC systems with refractive index (RI) [32,33]. Minerals were determined by inductively coupled plasma optical emis- sion spectroscopy (ICP-OES) [34]. The vitamins were measured with HPLC system by using various extraction methods [35-46]. Vitamin C includes both L-ascorbic acid Ldehydroascorbic acid. Niacin is reported as mcg that was not including tryptophan. Vitamin A active components; retinol and $\beta$-carotene were analysed by chromatographic methods. Vitamin A activity was then calculated as retinol equivalent activity (REA), using the following relationship:

$$
\text { Vit. A RAE = retinol }+1 / 12 \beta \text {-carotene }
$$

where all variables are expressed as $\mu \mathrm{g} 100 \mathrm{~g}^{-1}$ edible portion (EP).

\section{Results and discussion}

The findings of this study were categorised according to fruit groups such as stone fruits and pome fruits. The data produced in TürKomp were compared with data published in key 
Table IV. Proximate and carbohydrate composition of pome fruits (average values for the regions, $100 \mathrm{~g}^{-1}$ edible food).

\begin{tabular}{|c|c|c|c|c|c|c|c|c|c|c|c|c|c|c|}
\hline Fruits & Regions & $\begin{array}{c}\text { Water } \\
(\mathrm{g})\end{array}$ & $\begin{array}{l}\text { Enerc } \\
(\mathrm{kcal})\end{array}$ & $\begin{array}{l}\text { Ash } \\
\text { (g) }\end{array}$ & $\begin{array}{l}\text { Prot } \\
(\mathrm{g})\end{array}$ & $\begin{array}{l}\text { Fat } \\
\text { (g) }\end{array}$ & $\begin{array}{l}\text { Fibt } \\
(\mathrm{g})\end{array}$ & $\begin{array}{c}\text { Fibsol } \\
\text { (g) }\end{array}$ & $\begin{array}{c}\text { Fibins } \\
(\mathrm{g})\end{array}$ & $\begin{array}{c}\mathrm{CHO} \\
(\mathrm{g})\end{array}$ & $\begin{array}{c}\text { Sucs } \\
(\mathrm{g})\end{array}$ & $\begin{array}{l}\text { Glus } \\
\text { (g) }\end{array}$ & $\begin{array}{c}\text { Frus } \\
\text { (g) }\end{array}$ & $n$ \\
\hline \multirow{5}{*}{1} & $\mathrm{~A}$ & 84.2 & 44 & 0.38 & 0.59 & 0.16 & 3.59 & 0.24 & 3.35 & 11.1 & 0.12 & 2.47 & 4.13 & 2 \\
\hline & M & 83.4 & 52 & 0.31 & 0.58 & 0.15 & 3.19 & 0.24 & 2.95 & 12.4 & 0.57 & 2.42 & 5.39 & 2 \\
\hline & $\mathrm{MN}$ & 82.6 & 62 & 0.43 & 0.55 & 0.15 & 3.59 & 0.22 & 3.38 & 12.7 & 0.46 & 3.22 & 6.82 & 2 \\
\hline & Mean & 83.4 & 52 & 0.37 & 0.57 & 0.15 & 3.45 & 0.23 & 3.22 & 12.1 & 0.38 & 2.70 & 5.45 & 6 \\
\hline & $\mathrm{SD}$ & 1.6 & 13 & 0.07 & 0.15 & 0.03 & 0.35 & 0.03 & 0.33 & 1.5 & 0.51 & 0.57 & 1.54 & 6 \\
\hline \multirow{5}{*}{2} & Med & 85.2 & 52 & 0.31 & 0.41 & 0.12 & 3.31 & 0.33 & 2.98 & 10.7 & 2.95 & 0.01 & 0.01 & 2 \\
\hline & A & 85.1 & 54 & 0.28 & 0.56 & 0.09 & 3.21 & 0.24 & 2.97 & 10.9 & 4.70 & 0.01 & 0.01 & 2 \\
\hline & $\mathrm{MN}$ & 83.6 & 57 & 0.41 & 0.56 & 0.23 & 4.23 & 0.32 & 3.66 & 11.3 & 2.80 & 0.01 & 0.01 & 2 \\
\hline & Mean & 84.6 & 54 & 0.33 & 0.51 & 0.15 & 3.58 & 0.29 & 3.20 & 11.0 & 0.17 & 3.63 & 7.30 & 6 \\
\hline & $\mathrm{SD}$ & 1.9 & 7 & 0.08 & 0.55 & 0.08 & 0.74 & 0.16 & 0.48 & 1.0 & 0.20 & 1.46 & 1.35 & 6 \\
\hline \multirow{5}{*}{3} & $\mathrm{~A}$ & 85.3 & 52 & 0.37 & 0.42 & 0.55 & 1.85 & 0.56 & 1.29 & 11.5 & 8.40 & 0.01 & 0.02 & 3 \\
\hline & Med & 83.1 & 63 & 0.44 & 0.25 & 0.63 & 2.13 & 0.47 & 1.67 & 13.5 & 6.30 & 0.01 & 0.01 & 2 \\
\hline & MS & 84.7 & 56 & 0.32 & 0.36 & 0.60 & 1.43 & 0.39 & 1.05 & 12.7 & 5.70 & 0.02 & 0.01 & 2 \\
\hline & Mean & 84.5 & 56 & 0.38 & 0.35 & 0.58 & 1.81 & 0.48 & 1.33 & 12.4 & 1.53 & 2.12 & 4.18 & 7 \\
\hline & SD & 1.9 & 9.8 & 0.08 & 0.30 & 0.09 & 0.37 & 0.28 & 0.36 & 1.8 & 0.58 & 0.85 & 1.45 & 7 \\
\hline \multirow{5}{*}{4} & $\mathrm{~A}$ & 84.5 & 61 & 0.31 & 0.29 & 0.58 & 1.71 & 0.63 & 1.08 & 12.8 & 4.40 & 0.01 & 0.01 & 2 \\
\hline & M & 85.1 & 58 & 0.34 & 0.46 & 0.69 & 2.04 & 0.72 & 1.32 & 11.4 & 5.00 & 0.01 & 0.01 & 2 \\
\hline & Med & 85.2 & 57 & 0.39 & 0.25 & 0.57 & 1.99 & 0.52 & 1.47 & 11.7 & 3.80 & 0.01 & 0.01 & 2 \\
\hline & Mean & 84.9 & 59 & 0.35 & 0.33 & 0.61 & 1.91 & 0.62 & 1.29 & 12.0 & 2.22 & 2.03 & 6.94 & 6 \\
\hline & SD & 1.6 & 6.2 & 0.06 & 0.39 & 0.09 & 0.27 & 0.14 & 0.32 & 1.3 & 1.46 & 0.35 & 2.86 & 6 \\
\hline \multirow{5}{*}{5} & Med & 82.9 & 57 & 0.42 & 0.54 & 0.14 & 2.80 & 0.40 & 2.40 & 13.3 & 14.55 & 0.03 & 0.02 & 2 \\
\hline & $\mathrm{A}$ & 82.0 & 59 & 0.45 & 0.21 & 0.13 & 3.59 & 0.50 & 3.09 & 13.6 & 15.00 & 0.01 & 0.02 & 2 \\
\hline & $\mathrm{MN}$ & 80.8 & 64 & 0.48 & 0.31 & 0.17 & 3.31 & 0.52 & 2.80 & 14.9 & 16.10 & 0.01 & 0.02 & 2 \\
\hline & Mean & 81.9 & 60 & 0.45 & 0.35 & 0.14 & 3.23 & 0.47 & 2.76 & 13.9 & 0.14 & 3.26 & 4.68 & 6 \\
\hline & $\mathrm{SD}$ & 1.0 & 4.5 & 0.03 & 0.29 & 0.06 & 0.60 & 0.09 & 0.54 & 0.9 & 0.16 & 1.67 & 2.03 & 6 \\
\hline \multirow{2}{*}{6} & $\mathrm{M}$ & 72.5 & 143 & 1.33 & 0.61 & 11.5 & 9.70 & 0.33 & 9.37 & 4.36 & 8.50 & 0.06 & 0.13 & 2 \\
\hline & Mean & 72.5 & 143 & 1.33 & 0.61 & 11.5 & 9.70 & 0.33 & 9.37 & 4.36 & 0.02 & 0.04 & 0.13 & 2 \\
\hline
\end{tabular}

1: Pear winter 'Deveci', 2: Pear summer 'Santa Maria', 3: Apple winter 'Fuji', 4: Apple summer 'Gala', 5: Quince 'Eşme', 6: Avocado. MN: Mid,North, A: Aegean, M: Marmara, Med: Mediterranean, MS: Mid, Southern. Mean: Mean of individual samples, SD: Standard deviation, NA: Not available, EuroFIR/INFOODS component tagnames [13, 14]: WATER: water; ENERC: energy, total metabolisable; ASH: ash; PROT: protein; FAT: fat, total; FIBT/FIBTG: fibre, total dietary; FIBSOL: fibre, water-soluble; FIBINS: fibre, water-insoluble; CHO: carbohydrate; SUCS: sucrose; GLUS: glucose; FRUS: fructose.

Table V. Mineral and vitamin composition of plums (average values for the regions, $100 \mathrm{~g}^{-1}$ edible food).

\begin{tabular}{|c|c|c|c|c|c|c|c|c|c|c|c|c|c|c|c|c|}
\hline Fruits & Regions & $\begin{array}{c}\mathrm{Fe} \\
(\mathrm{mg})\end{array}$ & $\begin{array}{c}\mathrm{P} \\
(\mathrm{mg})\end{array}$ & $\begin{array}{c}\mathrm{Ca} \\
(\mathrm{mg})\end{array}$ & $\begin{array}{c}\mathrm{Mg} \\
(\mathrm{mg})\end{array}$ & $\begin{array}{c}\mathrm{K} \\
(\mathrm{mg})\end{array}$ & $\begin{array}{c}\mathrm{Na} \\
(\mathrm{mg})\end{array}$ & $\begin{array}{c}\mathrm{Zn} \\
(\mathrm{mg})\end{array}$ & $\begin{array}{l}\text { VitC } \\
(\mathrm{mg})\end{array}$ & $\begin{array}{l}\text { Thia } \\
\text { (mg) }\end{array}$ & $\begin{array}{l}\text { Ribf } \\
(\mathrm{mg})\end{array}$ & $\begin{array}{c}\mathrm{Nia} \\
(\mathrm{mg})\end{array}$ & $\begin{array}{l}\mathrm{VitB}_{6} \\
(\mathrm{mg})\end{array}$ & $\begin{array}{c}\text { VitA } \\
\text { (REA) }\end{array}$ & $\begin{array}{c}\text { Cartb } \\
(\mu \mathrm{g})\end{array}$ & $n$ \\
\hline \multirow{3}{*}{1} & A & 0.21 & 13 & 7 & 8 & 196 & 2 & 0.05 & 3.8 & 0.01 & 0.02 & 0.37 & 0.07 & 37 & 441 & 3 \\
\hline & Mean & 0.21 & 13 & 8 & 8 & 184 & 2 & 0.19 & 3.9 & 0.01 & 0.02 & 0.42 & 0.07 & 36 & 426 & 7 \\
\hline & SD & 0.11 & 6 & 4 & 2 & 39 & 0 & 0.34 & 1.45 & 0.00 & 0.01 & 0.07 & 0.03 & 17 & 203 & 7 \\
\hline \multirow{3}{*}{2} & Med & 0.13 & 23 & 9 & 10 & 160 & 4 & 0.14 & 4.5 & 0.01 & 0.02 & 0.31 & 0.04 & 17 & 204 & 2 \\
\hline & Mean & 0.12 & 20 & 8 & 7 & 140 & 3 & 0.11 & 4.8 & 0.01 & 0.01 & 0.33 & 0.05 & 18 & 216 & 7 \\
\hline & SD & 0.06 & 6 & 3 & 2 & 51 & 2 & 0.04 & 0.64 & 0.00 & 0.00 & 0.08 & 0.01 & 9 & 103 & 7 \\
\hline
\end{tabular}

1: Plum European plum group Stanley, 2: Plum Japan plum group Black Amber. A: Aegean, M: Marmara, Med: Mediterranean.

Mean: Mean of individual samples, SD: Standard deviation, NA: Not available.

EuroFIR/INFOODS component tagnames [13, 14]: FE: iron, total; P: phosphorus; CA: calcium; MG: magnesium; K: potassium; NA: sodium; ZN: zinc; VITC: vitamin C; THIA: thiamin, vitamin B-1; RIBF: riboflavin, vitamin B-2; NIA: niacin, preformed; VITB6: vitamin B-6, total; VITA: vitamin A; CARTB: beta-carotene. 
Table VI. Mineral and vitamin composition of cherries (average values for the regions, $100 \mathrm{~g}^{-1}$ edible food).

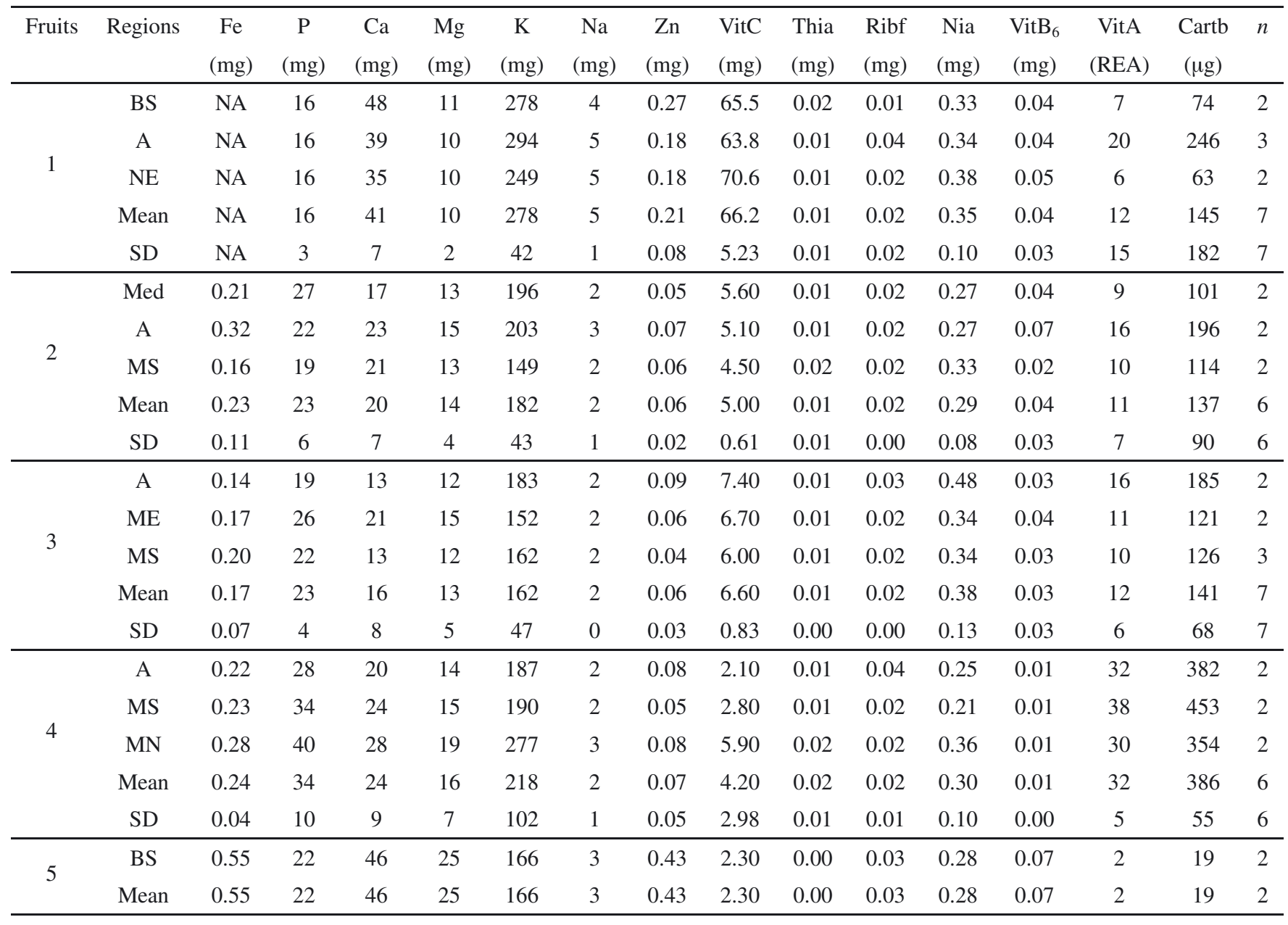

1: Cornelian cherry, 2: Sweet cherry, white, 'Starks Gold', 3: Sweet cherry, red, 'Ziraat 0900', 4: Sour cherry, 'Kütahya', 5: Laurel cherry. Mean: Mean of individual samples, SD: Standard deviation, NA: Not available.

EuroFIR/INFOODS component tagnames [13, 14]: FE: iron, total; P: phosphorus; CA: calcium; MG: magnesium; K: potassium; NA: sodium; ZN: zinc; VITC: vitamin C; THIA: thiamin, vitamin B-1; RIBF: riboflavin, vitamin B-2; NIA: niacin, preformed; VITB6: vitamin B-6, total; VITA: vitamin A; CARTB: beta-carotene.

national food composition databases (FCDBs of Denmark, UK and USA). The comparison of the range of data (min-max) produced in TürKomp for each fruit group and reported in other FCDBs is shown in table $X$. These databases were selected for comparison as they provide analytical data derived from laboratory analysis, as in TürKomp. The aim of this comparison was to capture any unforeseen errors in data production or unexpected differences in nutrient composition of fruits produced in Turkey. However, no significant difference was found between the food composition of fruits in TürKomp and other national FCTBs. It was seen that vitamin $\mathrm{C}$ data was slightly inconsistent among the FCTBs and TürKomp. This might be due to the sensitive nature of vitamin $C$, that gradually decreases following the harvest. Therefore, in food composition studies the rapid analysis of vitamin $\mathrm{C}$ concentration has paramount importance.

In the selected FCDBs, water-soluble fibers, insoluble fibers and individual sugars were not available for all fruit va- rieties. Data users can use the data available in TürKomp in place of the missing values. In TürKomp, cherries were sampled from 7 different agricultural regions (BS, A, NE, Med, $\mathrm{Ms}, \mathrm{MN}, \mathrm{ME})$. There are various types of cherries growing in Turkey of which the red sweet cherry has the most economic importance. Laurel cherry (Prunus lauracerasus) and Cornelian cherry (Cornus mas) species are mainly harvested from the wild, perceived as traditional medicines by the locals in Turkey and reported to be rich in antioxidants [48]. Therefore, these varieties were also selected in TürKomp in order to provide more food composition data on these regional varieties. Although its production is new in Turkey, avocado (Persea americana) was also selected in TürKomp due to its marketing potential, since it had recently become more popular in the country.

Cultivars, genetics, growing conditions, and geographical origin are the main factors that influence vitamin composition in fruits. Particularly, vitamin C content in foods depends on 
Table VII. Mineral and vitamin composition of almond, apricot, nectarine, and peach (average values for the regions, $100 \mathrm{~g}^{-1}$ edible food).

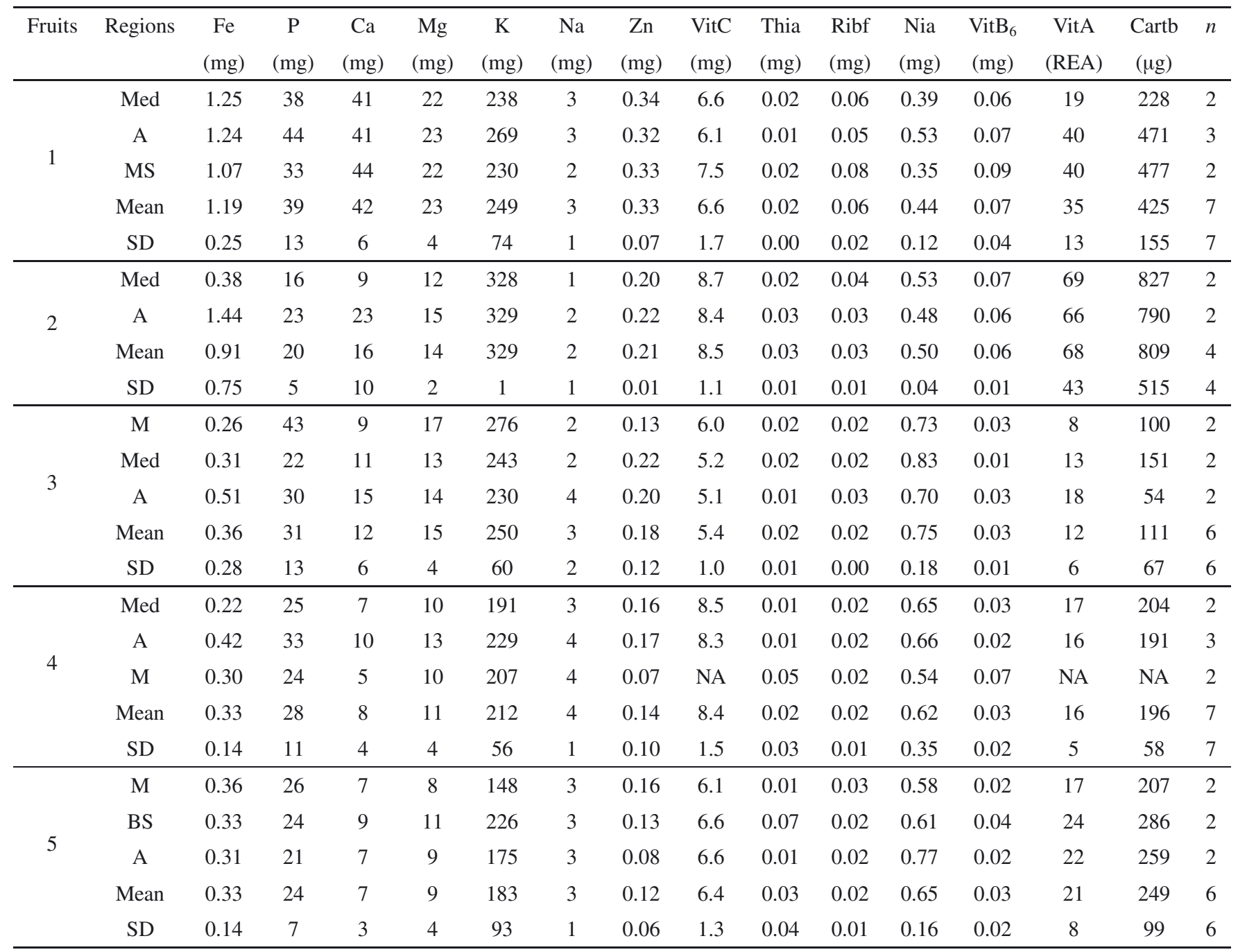

1: Green almond, 2: Apricot early season 'Tyrinthe', 3: Nectarine 'Big Top', 4: Peach early season, 5: Peach 'J. Hale'.

A: Aegean, M: Marmara, Med: Mediterranean, BS: Black Sea, MS: Mid, Southern.

Mean: Mean of individual samples, SD: Standard deviation, NA: Not available.

EuroFIR/INFOODS component tagnames [13,14]: FE: iron, total; P: phosphorus; CA: calcium; MG: magnesium; K: potassium; NA: sodium; ZN: zinc; VITC: vitamin C; THIA: thiamin, vitamin B-1; RIBF: riboflavin, vitamin B-2; NIA: niacin, preformed; VITB6: vitamin B-6, total; VITA: vitamin A; CARTB: beta-carotene.

genotypic variation, climatic conditions, intensity of light during growing, and postharvest handling [49]. As expected we have found significant regional variations in vitamin $C$ values mainly in Cornelian cherry, quince (Cydonia oblonga), apple 'Fuji', and sour cherry 'Kütahya' variations (standard devia-

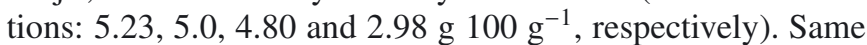
internal (genetic, etc.) and external (geographic conditions, etc.) factors affect biosynthetic pathway of pigments such as carotenoids and chlorophylls [50]. Therefore, comparison of beta-carotene in various regions, two to four fold differences were found in plums European plum group 'Stanley', Cornelian cherry, green almond, and quince 'Eşme'.
Agricultural regional data production is a useful approach for national FCDBs in order to get detailed information about the differences between the agricultural areas. This is because mean values and variations provide an overall composite of information about the food which can be used for various purposes such as agricultural policies. Food composition data produced over successive years can indicate trends in the food availability of the country that would inevitably inform national policies to safeguard agricultural production. The FCDBs may also support appropriate crop diversification policies to improve both agricultural income and production of nutritionally desirable foods, and estimate the need for important national food policies based on national food production 
Table VIII. Mineral and vitamin composition of pome fruits (average values for the regions, $100 \mathrm{~g}^{-1}$ edible food).

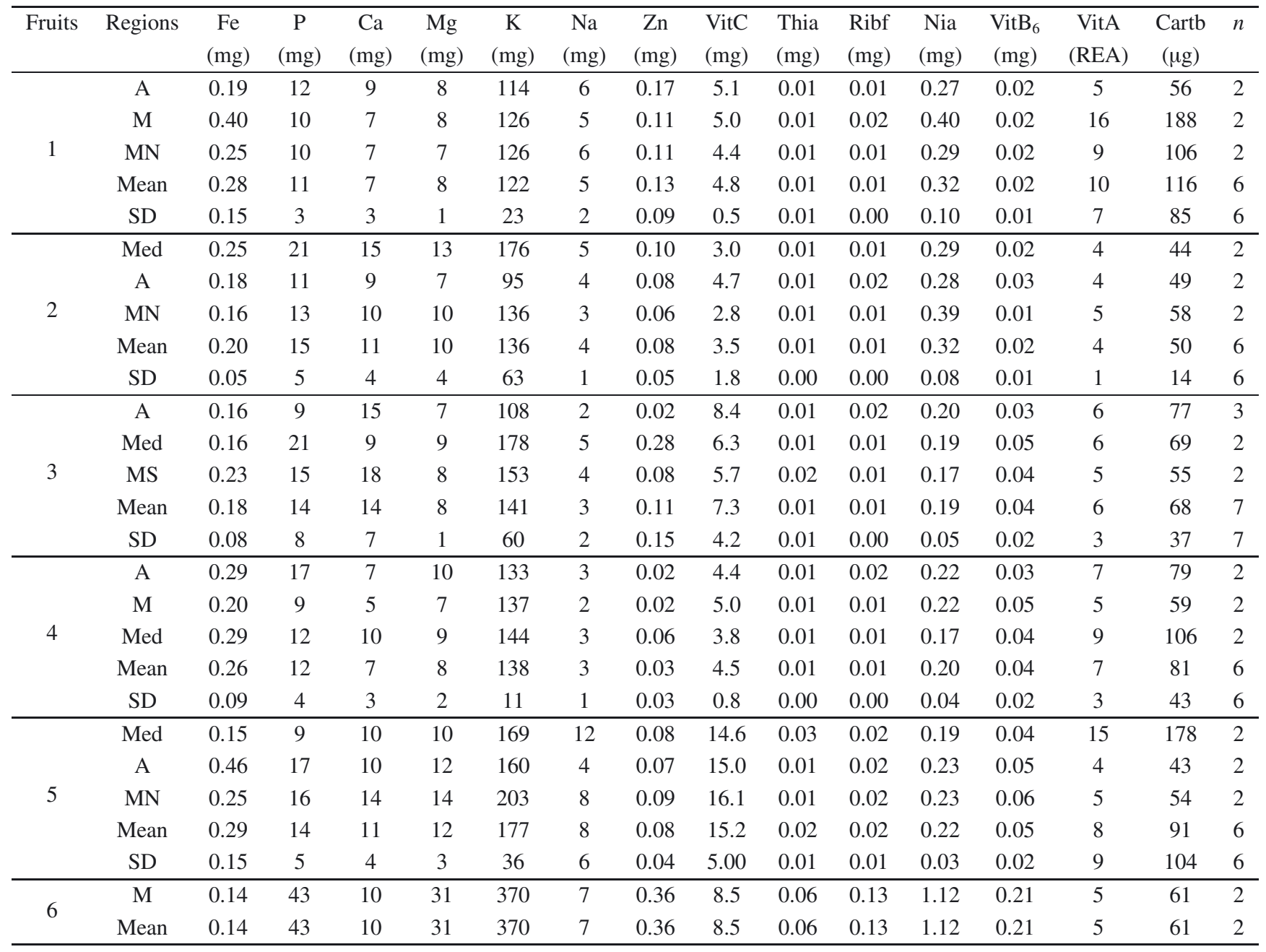

1: Pear winter 'Deveci', 2: Pear summer 'Santa Maria', 3: Apple winter 'Fuji', 4: Apple summer 'Gala', 5: Quince 'Eşme', 6: Avocado. MN: Mid,North, A: Aegean, M: Marmara, Med: Mediterranean, MS: Mid, Southern.

Mean: Mean of individual samples, SD: Standard deviation, NA: Not available.

EuroFIR/INFOODS component tagnames [13, 14]: FE: iron, total; P: phosphorus; CA: calcium; MG: magnesium; K: potassium; NA: sodium; ZN: zinc; VITC: vitamin C; THIA: thiamin, vitamin B-1; RIBF: riboflavin, vitamin B-2; NIA: niacin, preformed; VITB6: vitamin B-6, total; VITA: vitamin A; CARTB: beta-carotene.

quality [51]. Hence, national agricultural policies on fruit production in Turkey would benefit from successive data updates in TürKomp over the coming years as well as including more extensive varieties of fruits.

\section{Conclusion}

In this study, a FCDB, TürKomp, was established based on the analysis of a combination of both the micro and macronutrients of 18 core fruits widely consumed in Turkey. This is the first study developing a national FCDB in Turkey. A nationally represented database was needed in Turkey for various areas (e.g. public nutrition, agriculture and food trade). Authors believe this high standard FCDB expanded with systematic fu- ture updates may fill an important gap in food and nutrition research.

Acknowledgements. This study has been released within the 'Determination of National Food Composition and Formation of a Widely Available and Sustainable System' project (no: 107G208) and conducted under the TÜBITAK Support Programme for Research Projects of Public Institutions (KAMAG 1007). The authors would like to thank the project team and contractors responsible from sample handlings; T.R. Ministry of Agriculture and Livestock Food Control Laboratories of Izmir, Konya, Diyarbakır, Central Research Institute of Food and Feed Control Bursa, Central Research Institute of Fisheries Trabzon and T.R. Health Ministry; Public Health Laboratories of Erzurum, Istanbul, Izmir, Samsun, Head Department of Consumer Safety Ankara and TÜBITAK MRC Food Institute. The 
Table IX. The specifications of selected fruits and sampling characteristics in TürKomp.

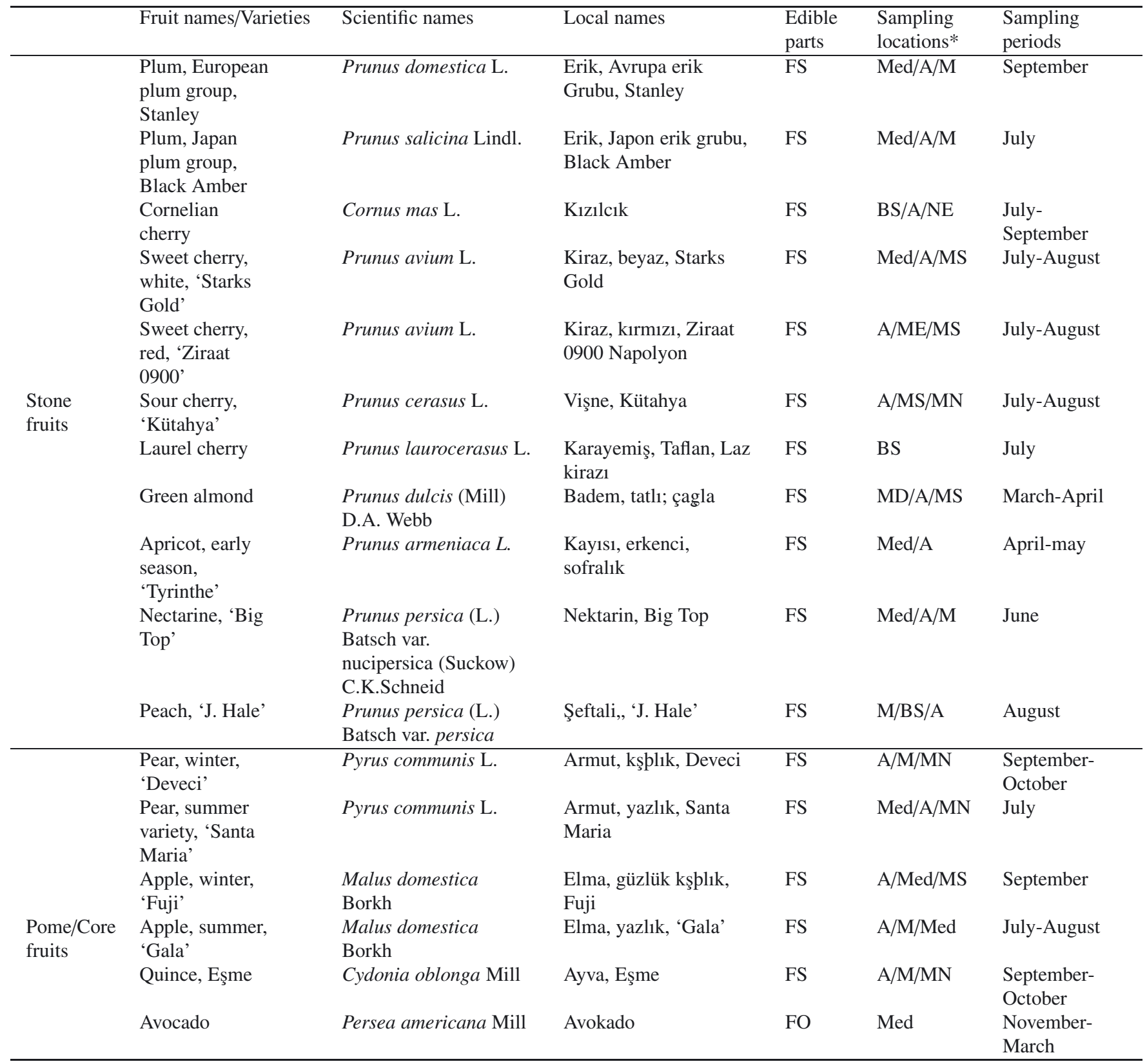

* Vegetation regions (provinces): MN: Mid,North (Ankara, Bilecik, Bolu, Çankırı, Çorum, Eskişehir, Kırpehir, Kütahya, Uşak, Yozgat, Kırıkkale, Düzce), A: Aegean (Aydın, Balıkesir, Burdur, Çanakkale, Denizli, Izmir, Isparta, Manisa, Muğla), M: Marmara (Bursa, Edirne, Istanbul, Kırklareli, Kocaeli, Sakarya, Tekirdağ, Yalova), Med: Mediterranean (Adana, Antalya, Gaziantep, Hatay, Içel, Kahramanmaraş, Kilis, Osmaniye), BS: Black Sea (Giresun, Gümüşhane, Kastamonu, Ordu, Rize, Samsun, Sinop, Trabzon, Zonguldak, Bayburt, Bartın, Karabük), ME: Middle East (Adıyaman, Amasya, Elazı̆̆, Malatya, Sivas, Tokat, Tunceli), NE: North East (Ağrı, Artvin, Erzincan, Erzurum, Kars, Ardahan, Iğdır), MS: Mid, Southern (Afyonkarahisar, Kayseri, Konya, Nevşehir, Niğde, Aksaray, Karaman).

FS: Flesh and skin, FO: Flesh only. 


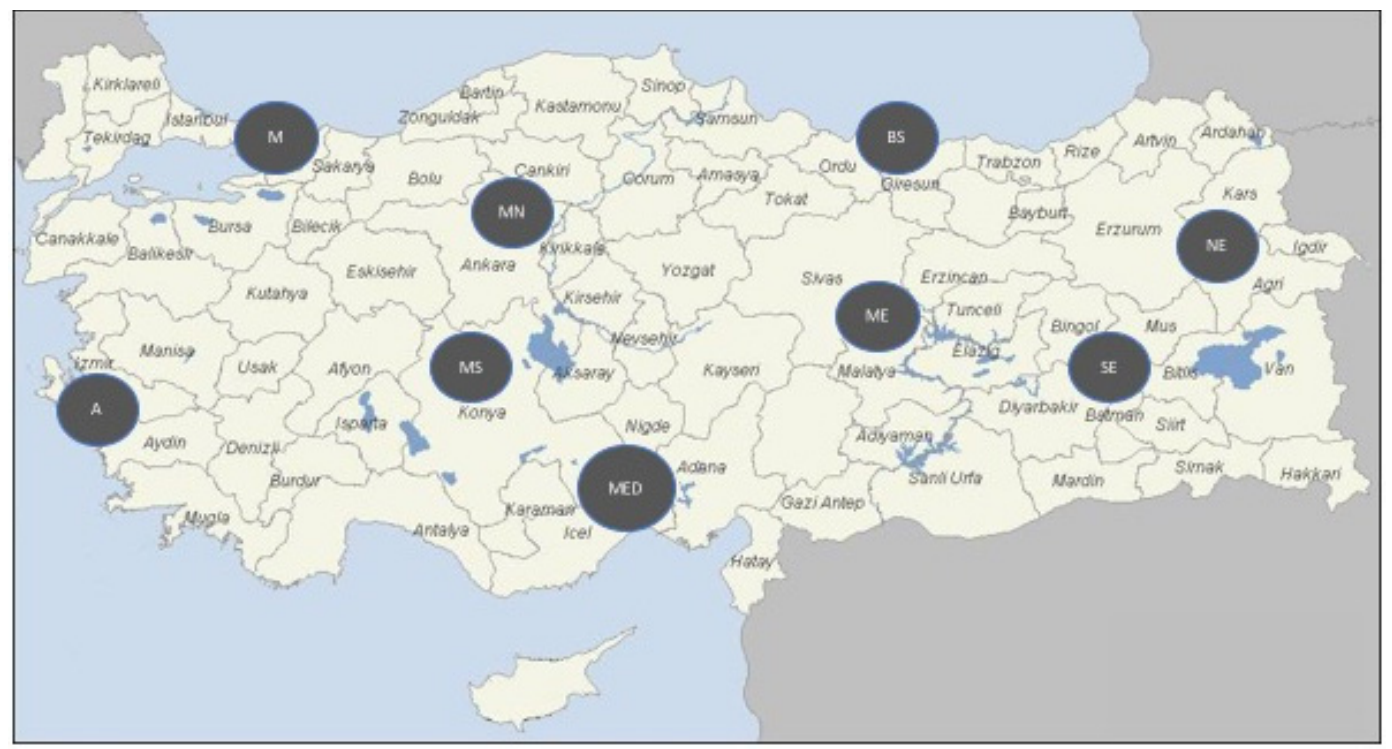

Figure 1. MN: Mid,North (Ankara, Bilecik, Bolu, Çankırı, Çorum, Eskişehir, Kırşehir, Kütahya, Uşak, Yozgat, Kırıkkale, Düzce), A: Aegean (Aydın, Balıkesir, Burdur, Çanakkale, Denizli, Izmir, Isparta, Manisa, Muğla), M: Marmara (Bursa, Edirne, Istanbul, Kırklareli, Kocaeli, Sakarya, Tekirdağ, Yalova), Med: Mediterranean (Adana, Antalya, Gaziantep, Hatay, Içel, Kahramanmaraş, Kilis, Osmaniye), BS: Black Sea (Giresun, Gümüşhane, Kastamonu, Ordu, Rize, Samsun, Sinop, Trabzon, Zonguldak, Bayburt, Bartın, Karabük), ME: Middle East (Adıyaman, Amasya, Elazığ, Malatya, Sivas, Tokat, Tunceli), NE: North East (Ağrı, Artvin, Erzincan, Erzurum, Kars, Ardahan, Iğdır), MS: Mid, Southern (Afyonkarahisar, Kayseri, Konya, Neşehir, Niğde, Aksaray, Karaman).

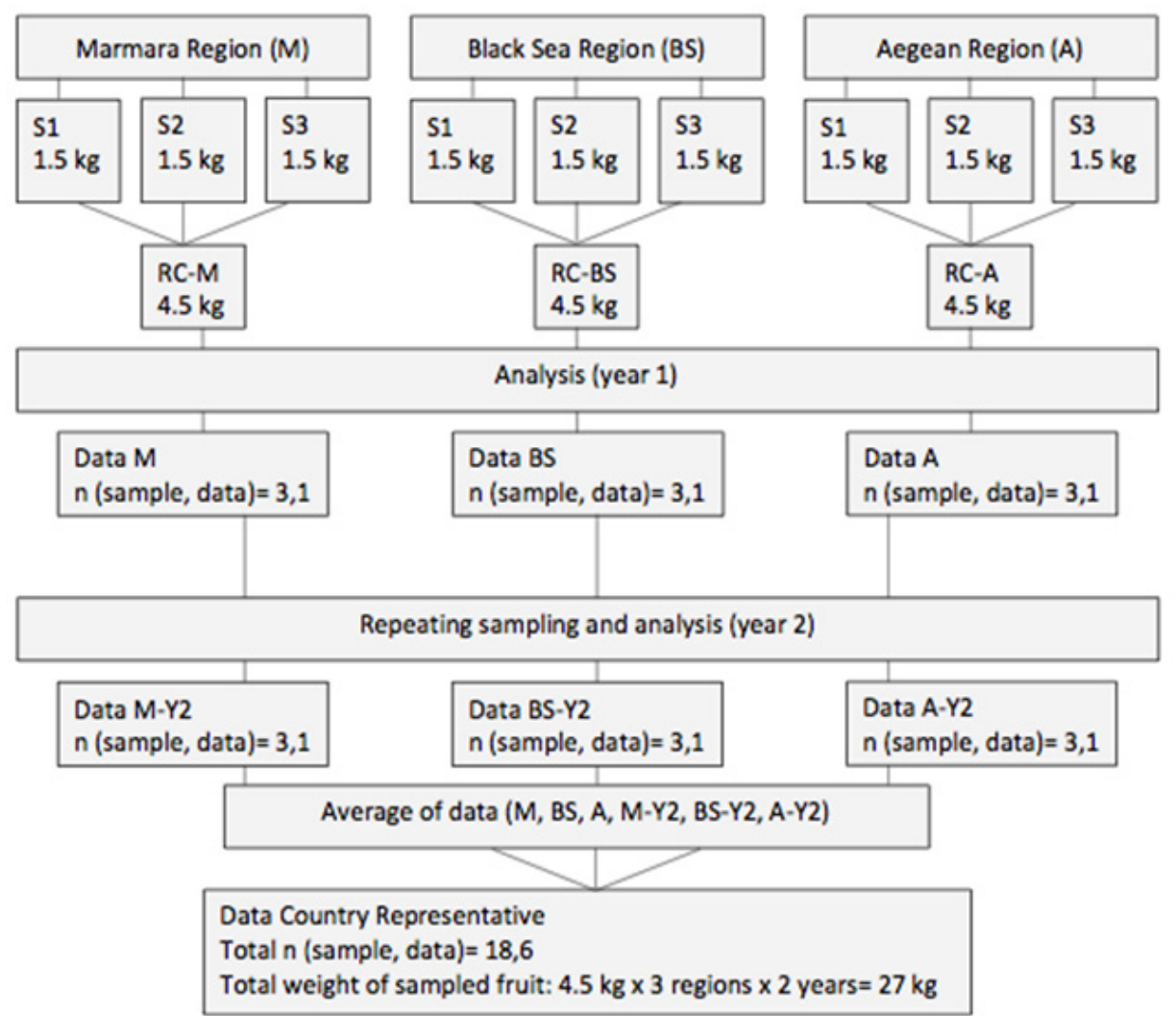

Figure 2. Sampling procedure of peach, 'J. Hale' in TürKomp. S: Sample, RC: Region composite, Y2: Year 2. 
Table X. The comparisons of the range of food composition data derived from TürKomp and other food composition databases (per $100 \mathrm{~g}$ edible portion). O: Data in other food composition databases (USDA, UK, Danish); T: Data produced for each fruit group within TürKomp.

\begin{tabular}{|c|c|c|c|c|c|c|c|c|c|c|}
\hline & \multicolumn{2}{|c|}{ Plum, raw } & \multicolumn{2}{|c|}{ Cherry, raw } & \multicolumn{2}{|c|}{ Sour cherry, raw } & \multicolumn{2}{|c|}{ Apricot, raw } & \multicolumn{2}{|c|}{ Nectarine, raw } \\
\hline & $\mathrm{O}$ & $\mathrm{T}$ & $\mathrm{O}$ & $\mathrm{T}$ & $\mathrm{O}$ & $\mathrm{T}$ & $\mathrm{O}$ & $\mathrm{T}$ & $\mathrm{O}$ & $\mathrm{T}$ \\
\hline Prot $(\mathrm{g})$ & $0.50-0.70$ & $0.08-0.25$ & $0.90-1.40$ & $0.58-0.96$ & 1 & $0.53-0.65$ & $0.90-1.4$ & $0.25-0.28$ & $1.06-1.40$ & $0.16-0.27$ \\
\hline Cartb $(\mu \mathrm{g})$ & $80.0-376$ & $103-549$ & $25.0-38.0$ & $63.0-246$ & 770 & $354-453$ & $405-1566$ & $790-827$ & $114-150$ & $54.0-151$ \\
\hline B1 (mg) & $0.02-0.05$ & $0.01-0.01$ & $0.03-0.03$ & $0.01-0.02$ & 0.03 & $0.01-0.02$ & $0.03-0.03$ & $0.02-0.03$ & $0.02-0.03$ & $0.01-0.02$ \\
\hline B2 (mg) & $0.03-0.03$ & $0.01-0.02$ & $0.03-0.03$ & $0.02-0.03$ & 0.04 & $0.02-0.04$ & $0.03-0.04$ & $0.03-0.04$ & $0.03-0.04$ & $0.02-0.03$ \\
\hline B6 (mg) & $0.03-0.05$ & $0.04-0.07$ & $0.05-0.05$ & $0.02-0.07$ & 0.044 & $0.01-0.01$ & $0.05-0.05$ & $0.06-0.07$ & $0.03-0.03$ & $0.01-0.03$ \\
\hline VitC (mg) & $4.0-9.5$ & $3.0-5.2$ & $7.0-11.0$ & $4.5-7.4$ & 10 & $2.1-5.9$ & $10.0-11.0$ & $8.4-8.7$ & $5.4-37$ & $5.1-6.0$ \\
\hline $\mathrm{Ca}(\mathrm{mg})$ & $6.0-13.0$ & $6.0-10.0$ & $13.0-20.8$ & $13.0-23.0$ & 16 & $20.0-28.0$ & $13.0-15.0$ & $9.0-23.0$ & $6.0-7.0$ & $9.0-15.0$ \\
\hline $\mathrm{Fe}(\mathrm{mg})$ & $0.14-0.40$ & $0.07-0.29$ & $0.20-0.40$ & $0.14-0.32$ & 0.32 & $0.22-0.28$ & $0.39-0.50$ & $0.38-1.44$ & $0.28-0.40$ & $0.26-0.51$ \\
\hline $\mathrm{Mg}(\mathrm{mg})$ & $5.0-8.0$ & $6.0-10.0$ & $8.0-11.0$ & $12.0-15.0$ & 9 & $14.0-19.0$ & $10.0-11.0$ & $12.0-15.0$ & $9.0-10.0$ & $13.0-17.0$ \\
\hline $\mathrm{n}$ & $\mathrm{O}$ & $\mathrm{T}$ & $\mathrm{O}$ & $\mathrm{T}$ & $\mathrm{O}$ & $\mathrm{T}$ & $\mathrm{O}$ & $\mathrm{T}$ & $\mathrm{O}$ & $\mathrm{T}$ \\
\hline Prot $(\mathrm{g})$ & $0.70-1.0$ & $0.71-1.06$ & $0.30-0.36$ & $0.41-0.59$ & $0.26-0.60$ & $0.25-0.46$ & $0.30-0.40$ & $0.21-0.54$ & $1.90-2.00$ & 0.61 \\
\hline Fibt (g) & $1.5-1.7$ & $1.3-1.9$ & $1.6-3.2$ & $3.2-4.2$ & $1.3-2.4$ & $1.4-2.1$ & 1.9-1.9 & $2.8-3.6$ & 3.4.6.7 & 9.7 \\
\hline Cartb $(\mu \mathrm{g})$ & $114-162$ & $191-286$ & $14.0-65.0$ & $44.0-188$ & $14.0-27.0$ & 55-106 & $0-24.0$ & $4.0-15.0$ & $16-28$ & 61 \\
\hline B1 (mg) & $0.02-0.02$ & $0.01-0.07$ & $0.01-0.03$ & $0.01-0.01$ & $0.02-0.04$ & $0.01-0.02$ & $0.02-0.02$ & $0.01-0.03$ & $0.07-0.1$ & 0.06 \\
\hline B2 (mg) & $0.03-0.06$ & $0.02-0.03$ & $0.02-0.04$ & $0.01-0.02$ & $0.01-0.04$ & $0.01-0.02$ & $0.02-0.03$ & $0.02-0.02$ & $0.13-0.18$ & 0.128 \\
\hline Nia (mg) & $0.60-0.80$ & $0.54-0.77$ & $0.20-0.30$ & $0.27-0.40$ & $0.09-0.10$ & $0.17-0.22$ & $0.20-0.20$ & $0.19-0.23$ & $1.10-1.70$ & 1.12 \\
\hline B6 (mg) & $0.02-0.03$ & $0.02-0.07$ & $0.02-0.04$ & $0.01-0.03$ & $0.04-0.07$ & $0.03-0.05$ & $0.04-0.04$ & $0.04-0.06$ & $0.30-0.40$ & 0.211 \\
\hline VitC (mg) & $6.6-31$ & $6.1-8.5$ & $3.0-4.94$ & $2.8-5.1$ & $4.6-10.0$ & $3.8-8.4$ & $15.0-15.0$ & $14.6-16.1$ & $6.0-10.0$ & 8.5 \\
\hline
\end{tabular}

$\mathrm{n}=$ Number of data (as for TürKomp; it is the number of data produced for each regional sample).

EuroFIR/INFOODS component tagnames [13, 14]: PROT: protein; FIBT/FIBTG: fibre, total dietary; FE: iron, total; CA: calcium; MG: magnesium; K: potassium; VITC: vitamin C; THIA: thiamin, vitamin B-1; RIBF: riboflavin, vitamin B-2; NIA: niacin, preformed; VITB6: vitamin B-6, total; CARTB: beta-carotene.

authors would like to thank Prof. Dr. Turgut Yeşiloğlu, Prof. Dr. Nebahat Sarı, Assistant Prof. Bilge Yıldırım from Çukurova University Faculty of Agriculture Department of Horticulture, for their contributions to sample protocols. The authors would like to thank TÜBITAK MRC FI nutrition laboratory R\&D technicians (also project stuff); şenol Erdoğan, Tamer Işık, and Fuat Arslan for their hard works. The authors also would like to thank Nishat Mirza for her contribution to final grammar control of this paper.

\section{References}

[1] Yıldıztugay E., BağcıY., Küçükoduk M., Endemic plants of Baparakavak and environs (Konya, Turkey), Proceedings of 5th Balkan Botanical Congress, Belgrade, Serbia, 2009, pp. 23-24.
[2] TUIK, News relase, 2014. Available from: http://www.turkstat.gov.tr/PreHaberBultenleri.do?id=13513 - 3 2013- 1442014.

[3] Theuwissen E., Mensink R.P., Water soluble fibers and cardiovascular disease, Physiol. Behav. 94 (2008) 285-292.

[4] TUIK, Bitkisel üretim istatistikleri veri tabanı, 2015. Available from: http://www.tuik.gov.tr/PreTablo.do?alt_id=1001

[5] TBSA, Turkiye beslenme ve saglik arastirmasi, T.C. Saglik Bakanligi, Hacettepe Universitesi, Turkey, 2014. Available from: www.sagem.gov.tr/TBSA_Beslenme_Yayini.pdf.

[6] Greenfield H., Southgate D.A.T., Food composition data, production, management, FAO Publishing Service, 2nd ed., 1-225, Rome, Italy, 2003.

[7] Biringen Löker G., Özkoç S.Ö., Amoutzopoulos B., Yaman M., Akkup S., SanlıF., Küçük, F., Establishing a food composition database for Turkey besed on European standarts, Nutr. Bull. 36 (2011) 254-258. 
[8] Pehrsson P.R., Haytowitz D.B., Holden J.M., Perry C.R., Beckler D.G., USDA's national food and nutrient analysis program: Food sampling, J. Food Comp. Anal. 13 (2000) 379-389.

[9] Galeazzi M.A.M., Lima M.D., Colugnati F.A.B., Padovani R.M., Rodriguez-Amaya D.B., Sampling plan for the Brazilian TACO project, J. Food Comp. Anal. 15 (2002) 499-505.

[10] Haytowitz D.B., Pehrsson P.R., Holden J.M.,The identification of key foods for food composition research, J. Food Comp. Anal. 15 (2002) 183-194.

[11] Holden J.M., Food comp course lecture notes: Sampling, WUEuroFIR, Wageningen, The Netherlands, 2005.

[12] Haytowitz D.B., Pehrsson P.R., Holden J.M., The national food and nutrient analysis program: A decade of progress, J. Food Comp. Anal. 21 (2008) S94-S102.

[13] Møller A., Unwin I.D., Ireland J., Roe M.A., Becker W., Colombani P., The EuroFIR Thesauri 2008, EuroFIR, Norwich, 2008. Available from: http://www.fao.org/uploads/ media/EuroFIR_Thesauri_2008_02.pdf

[14] FAO, Tagnames for food components, International Network of Food Data Systems (INFOODS), 2016. Available from: http://www.fao.org/infoods/infoods/standards-guidelines/foodcomponent-identifiers-tagnames/en/

[15] Watt B.K., Merrill A.L., Composition of foods raw, processed, prepared, US Department of Agriculture, Agriculture Handbook 8, 1963.

[16] Codex, Guidelines on Nutrition Labelling, CAG/GL-1985, 2011.

[17] AOAC, Solids (total) and moisture in flour air oven method 990.19, AOAC International Official Methods of Analysis, 1993.

[18] AOAC, Solids (total) and moisture in milk air oven method 990.20, AOAC International Official Methods of Analysis, 2010a.

[19] AOAC, Solids (total) and moisture in flour air oven method 925.10, AOAC International Official Methods of Analysis, $2010 \mathrm{~b}$

[20] AOAC, Ash of sugars and syrups 900.02, AOAC International Official Methods of Analysis, 2002.

[21] AOAC, Ash of flour 923.03, AOAC International Official Methods of Analysis, 2010c.

[22] Jones D.B., Factors for converting percentages of nitrogen in foods and feeds into percentages of protein, United States Department of Agriculture Washington D.C., Circular No 183, August 1931, Rev. February, USA, 1941.

[23] AOAC, Microchemical determination of nitrogen-micro Kjeldahl method 960.52, AOAC International Official Methods of Analysis, 2010d.

[24] TS, Türk StandartlarıTS 765, Yağli tohum küspelerinde dietil eter ile ekstrakte edilebilen yağ tayini (16.07.1969), Ankara, Turkey, 1969.

[25] TS, Türk StandartlarıTS 1745 ISO 1444, Et ve et mamulleriserbest yağ muhtevasitayini (07.04.1999), Ankara, Turkey, 1999.

[26] TS, Türk Standartları, TS 2664, Konserve-bitkisel sıvıYağli barbunya pilaki (12.06.2007), Ankara, Turkey, 2007.

[27] AOAC, Total soluble and insoluble dietary fiber in foodsenzymatic-gravimetric method MES-TRIS buffer 991.43, AOAC International Official Methods of Analysis, 1994.

[28] Megazym, Megazyme International, Total dietary fibre assay procedure, Ireland Limited, 2005.

[29] DIN, Analysis of honey, Determination of the content of saccharides; fructose, glucose, saccharose, turanose and maltose, HPLC method, DIN 10758, 1997-05, 1997.
[30] Richmond M.L., Brandao S.C.C., Gray J.I., Markakis P., Stine C.M., Analysis of simple sugars and sorbitol in fruit by highperformance liquid chromatography, J. Agric. Food Chem. 29 (1981) 4-7.

[31] Erkan N., Özden Ö., Proximate composition and mineral contents in aqua cultured sea bass (Dicentrarchus labrax), sea bream (Sparus aurata) analyzed by ICP-MS, Food Chem. 102 (2007) 721-725.

[32] Finglas P.M., Faulks R.M., The HPLC analysis of thiamin and riboflavin in potatoes, Food Chem. 15 (1984) 37-44.

[33] Reyes E.S.P., Subryan L., An improved method of simultaneous HPLC assay of riboflavin and thiamine in selected cereal product, J. Food Comp. Anal. 2 (1989) 41-47.

[34] Sampson D.A., Eoff L.A., Yan X.L., Lorenz K., Analysis of free and glycosylated vitamin B6 in wheat by HPLC, Cereal Chem. 72 (1995) 217-221.

[35] Tompson C.O., Trenerry V.C., A rapid method for the determination of total L-ascorbic acid in fruits and vegetables by miceller electrokinetic capillary chromatography, Food Chem. 53 (1995) 43.

[36] Konings E.J.M., Roomans H.H.S., Evaluation and validation of an LC method for the analysis of carotenoids in vegetables and fruit, Food Chem. 59(4) (1997) 599-603.

[37] Esteve M.J., Farre R., Frigola A., Garcia-Cantabella J.M., Determination of vitamin B6 (PM, PN, PL) in pork meat and pork meat products by liquid chromatography, J. Chromatogr. A 795 (1998) 383-387.

[38] Gökmen V., Kahraman N., Demir N., Acar J., Enzymatically validated liquid chromatographic method for the determination of ascorbic and dehydroascorbic acids in fruit and vegetables, J. Chromatogr. A 881(1-2) (2000) 309-316.

[39] Esteve M.J., Farre R., Frigola A., Cantabella J.M.G., Simultaneous determination of thiamine and riboflavin in mushrooms by liquid chromatography, J. Agric. Food Chem. 49 (2001) 1450-1454.

[40] Sallin-Rose C., Blake C.J., Genaoud D., Tagliaferri E.G., Comparison of microbiological and HPLC fluorescence detection methods for determination of niacin in fortified food products, Food Chem. 73 (2001) 473-480.

[41] Ndaw S., Bergaentzle M., Aoude-Werner D., Hasselmann C., Enzymatic extraction prodedure for the liquid chromatographic determination of niacin in foodstuffs, Food Chem. 78 (2002) 129-134.

[42] Brause A.R., Woollard D.C., Indky H.E., Determination of total vitamin $\mathrm{c}$ in fruit juices and related products by liquid chromatography interdisciplinary study, J. AOAC Int. 86 (2003) 367.

[43] Kall M.A., Determination of total vitamin B6 in foods by isocratic HPLC: A comparison with microbiological analysis, Food Chem. 82(2) (2003) 315-327.

[44] Tang X., Cronin D.A., Brunton N.P., A simplified approach to the determination of thiamine and riboflavin in meats using reverse phase HPLC, J. Food Comp. Anal. 19 (2006) 831-837.

[45] Eittenmiller R.R., Lin Ye W.O., Landen Jr., Vitamin analysis for food the health and food sciences, CRC Press, New York, Second Edition 3-81, 2008. 
[46] DS/EN, Foodstuffs determination of niacin by HPLC. DS/EN 15652 (E) 2009-06-23, 2009.

[47] Hefni M., Veronica O., Tabekha M., Witthoft C., Folate content in foods commonly consumed in Egypt, Food Chem. 121 (2010) 540-545.

[48] Capanoglu E., Boyacioglu D, de Vos R.C.H., Hall R.D., Beekwilder J., Procyanidins in fruit from Sour cherry (Prunus cerasus) differ strongly in chainlength from those in Laurel cherry (Prunus lauracerasus) and Cornelian cherry (Cornus mas), J. Berry Res. 1 (2011) 137-146.
[49] Lee S.K., Kader A.A., Preharvest and postharvest factors influencing vitamin $\mathrm{C}$ content of horticultural crops, Postharvest Biol. Technol. 20 (2000) 207-220.

[50] Ferreira de Faria A., Hasegawa P.N., Chagas E.A., Pio R., Purgatto E., Mercadante A.Z., Cultivar influence on carotenoid composition of loquats from Brazil, J. Food Comp. Anal. 22 (2009) 196-203.

[51] Latham M.C., Human nutrition in the developing world, FAO, Rome, 1997. Available from: http://portals.wi.wur.nl/foodnut/ latham/Lathamtoc.htm\#TopOfPage

Cite this article as: Gül Biringen Löker, Mustafa Yaman, Senem Akkuş Çevikkalp, Nuran Başaran, Çağrı Özalp, Şebnem Gelgel Ünlü, Demet Demirci Gültekin, Savaş Çalık, Murat Öztürk, Hasan Altay, Ilknur Türker, Hacer Ceyhan, Azize Alayli Güngör, Birdem Amoutzopoulos. Food composition of fruits in Turkey: research outputs from the new Turkish Food Composition Database, TürKomp. Fruits 71 (2016) 419-431. 